Conclusions: Although there appears to be a trend toward a worsening of cardiac function with clozapine treatment, further investigations need to be carried out taking into account confounding factors that are known to be implicated in cardiac dysfunction. Establishing a clearer understanding of the link between the two will help patients and clinicians balance the risk of cardiac problems and improved psychopathology and help to institute cardiac monitoring guidelines for patients treated with clozapine.

\section{Gamma synchrony in first-episode psychosis}

\section{G Flynn ${ }^{1,2}$, D Alexander ${ }^{1,3}$, C Galletly ${ }^{1,4}$, E Gordon ${ }^{1,3}$, $A H^{1,5}, T$ Whitford ${ }^{1,6}$, L Williams ${ }^{1,6}$, W Wong ${ }^{1,7}$, P Boord ${ }^{1,6}$}

'Brainnet Schizophrenia Research Group; ${ }^{2}$ SSWAHS Early Psychosis Intervention Program; ${ }^{3}$ Brain Resource Company; ${ }^{4}$ Adeleide University; ${ }^{5}$ The University of Sydney; ${ }^{6}$ Brain Dynamics Centre; and ${ }^{\top}$ The Early Psychosis Intervention Program Liverpool Hospital, Australia

Background: This project will compare gamma synchrony in 55 subjects with recent onset psychosis and 110 age-, sex- and education-matched controls. An auditory oddball and a visual working memory paradigms were used, to explore the hypothesis that disturbed 'binding' in psychosis is not limited to auditory processing.

Methods: The EEG data were analyzed using a measure of phase synchrony, described in detail elsewhere (Haig et al. 2000). There were two important differences in the present work. First, multiple frequencies were assessed, rather than simply $40 \mathrm{~Hz}$. Second, a 512-ms fast Fourier transform window was used to estimate the phases at a given frequency, rather than $256 \mathrm{~ms}$. This provided an improved frequency resolution $(\sim 2 \mathrm{~Hz})$, at the expense of temporal resolution. The phase synchrony was estimated for six frequency bands between 35 and $45 \mathrm{~Hz}$ at $10-\mathrm{ms}$ intervals.

Results: Multiple analyses of covariance (controlling for age) showed significant excesses of gamma synchrony in the psychosis group for both paradigms with different topographical distribution in each. In general, there were more marked differences in the auditory paradigm, a dominant frontal and left-sided abnormality in both, with elevated synchrony posteriorly in the visual paradigm.

Conclusion: Elevated synchrony in both paradigms supports the potential role of abnormal assembly formation as a generalized mechanism responsible for the production of psychotic symptoms.

\section{Duration of untreated psychosis: the relative contribution of individual vs. community factors}

\author{
A Frost' ${ }^{1}$, Carr $^{2}$, S Catts ${ }^{3}$, B 0'Toole ${ }^{4}$
}

'Royal Brisbane and Women's Hospital; ${ }^{2}$ Neuroscience Institute of Schizophrenia and Allied Disorders (NISAD); ${ }^{3}$ Hospital and Community Psychiatry, University of Queensland; and ${ }^{4}$ ANZAC Institute, The University of Sydney, Sydney, Australia

Background: Although some debate exists in the literature, there is a general consensus that patients with a longer duration of untreated psychosis (DUP) have poorer health outcomes than those treated early. Two recent meta-analyses have established this relationship as being of moderate effect size. Recent research has focused on reducing DUP through either a community approach or a 'at-risk' individuals approach. However, it is not currently established in the literature the relative contributions of individual vs. community factors predicting DUP.

Methods: The present study examined an existing research cohort of 456 Early Psychosis (EP) patients, from 19 mental health teams. The DUP of 326 of these patients had been assessed by the clinician. Multilevel modeling was used to establish the relative contribution of service level variables, and patient level variables.

Results: The initial null model showed that the service level accounted for $0.54 \%$ of potentially explainable variance in the total model; this was not significant $(P=0.36)$. The remaining $99.46 \%$ of variance was accounted for by the patient level. An exploratory analysis of individual factors showed that item 7 of the HoNOS (Depressed Mood) had a significant positive relationship to DUP $(P=0.01)$, while item 10 (problems with ADLs) had a negative relationship approaching significance $(P=0.06)$.

Conclusions: While other individual predictive relationships need to be tested, this study identifies depression as a candidate risk factor for prolonged DUP. Importantly, this research also highlights the central importance of individual factors over community factors in producing treatment delays.

\section{Multimodal imaging of the mismatch negativity deficit in schizophrenia}

\section{R Fulham', P Michie', P Ward', M Hughes', U Schall' \\ 'Centre for Brain and Mental Health Studies, Newcastle University; ${ }^{2}$ School of Behavioural Sciences, Newcastle University; and ${ }^{3}$ Schizophrenia Research Unit, Liverpool Hospital, Liverpool, Australia}

Background: Mismatch negativity (MMN) is an electrophysiological response to novel auditory stimuli. This project examines the developmental time course 
of the MMN deficit in schizophrenia and its neurological basis.

Method: EEG and functional magnetic resonance imaging (fMRI) data were obtained from first-episode and chronic patients with schizophrenia, and matched controls. A duration-deviant MMN paradigm was used in which participants heard an unattended random series of tones consisting of standards $(92 \%, 50 \mathrm{~ms})$ and deviants $(8 \%, 100 \mathrm{~ms})$. High-resolution MMNs were obtained as the difference between deviant and standard ERPs. Cortically constrained LORETA current source density analysis was performed using realistic head models.

Results: A reduction in MMN amplitude was seen in younger patients ( 40 years). This is consistent with our previous findings. Current source density analysis of the early phase of the MMN suggests that the major cortical generator of the MMN lies in the superior temporal gyrus (STG) as expected. The latter phase of the MMN engages more anterior cortical regions including premotor cortex. Patients show reduced activity in STG but increased activity in right premotor cortex. fMRI analysis showed that patients have greater activation in the insula and premotor cortex, whereas controls show greater activation in middle frontal gyrus.

Conclusions: The results are consistent with a deficit that onsets early in the disorder, that is associated with substantially reduced processing within auditory cortex and that leads to different patterns of activation in frontal cortical regions in patients compared with controls.

\section{Investigation of circadian disruption in bipolar disorder}

\author{
J Fullerton', A Heath', P MitchelI², P Schofield \\ Prince of Wales Medical Research Institute; and 'Mood Disorders Unit, \\ The Black Dog Institute, Sydney, Australia
}

Background: There is a growing body of evidence implicating circadian disruption as a possible mechanism underlying the pathogenesis of bipolar disorder. The circadian clock is entrained by changes in our external environment and regulates many of our bodies functions, including our sleep-wake cycle, metabolism, thermoregulation and blood pressure. While the measurement of circadian rhythmicity in mice is relatively straight forward through automated recording of home cage activity, the measurement of circadian function/ disruption in humans is more difficult and costly, requiring each patient to undergo actigraphy in a sleep laboratory over a period of several days.

Methods: To collect data on a scale sufficient for genetic studies, a more efficient method of monitoring circadian function in humans is required to determine whether circadian disruption is involved in the primary pathogenesis of bipolar disorder or whether symptomatic sleep disruption is a secondary defect. We are investigating the role of circadian disruption in bipolar disorder, by genetic association, and rhythmic gene expression using immortalized B lymphocytes from a cohort of Australian bipolar families.

Results: Circadian timing in immortalized B lymphocytes is synchronized by serum shock, and circadian gene expression ensues for at least $56 \mathrm{~h}$ post synchronization.

Conclusions: Peripheral tissues, including immortalized blood cells, can be used as a cheap and higher throughput method to measure circadian output in humans and will be a useful adjunct to elucidating the molecular mechanisms underlying bipolar disorder.

\section{Silence is golden? Improving cognitive performance in schizophrenia}

\section{Galletly', M Proeve ${ }^{2}$, C Field ${ }^{2}$, K Harvey ${ }^{2}$}

University of Adelaide; and 2University of South Australia, Adelaide, Australia

Cognitive impairment is a common and disabling symptom of schizophrenia and has adverse effects on functional outcome. Improvement in cognitive function has now been identified as a major target in the treatment of schizophrenia. Cognitive remediation strategies where people with schizophrenia are required to speak aloud while performing learning tasks have shown some success. The present study compared performance on tasks requiring parallel vocalization with task performance when the person did not speak aloud. Results showed selective benefits of verbal strategy on a neuropsychological task requiring multiple executive functions but not on tasks requiring simpler single-component cognitive functions.

\section{Can consent be uninformed? Suggested reform of sexual offences against persons with mental impairment}

\section{Graydon}

Medicine, Nursing and Health Sciences, Murdoch University, WA, Australia

In R v Morgan (1970), the Supreme Court of Victoria stated that for incapacity to consent to be proved, it must be shown that a person 'has not sufficient knowledge or understanding to comprehend (a) that what is 